\title{
Spontaneous spinal subarachnoid hemorrhage associated with subdural hematoma at different spinal levels
}

\author{
Yousuke Kakitsubata • Stavroula J. Theodorou • \\ Daphne J. Theodorou • Yuko Miyata • Yasunori Ito • \\ Yasuhiro Yuki • Koichi Honbu • Toyo Maehara
}

Received: 18 October 2008 / Accepted: 19 December 2008 / Published online: 28 January 2009

(C) The Author(s) 2009. This article is published with open access at Springerlink.com

\begin{abstract}
We describe the clinical features and MRimaging findings of spontaneous spinal subarachnoid hemorrhage located in the lumbar spine associated with subdural hematoma at a higher, thoracic level in a 66-yearold man without neurological deficit. The sequential MRimaging changes of hemorrhage at various stages in its evolution are portrayed. The possible pathogenetic mechanism for these very unusual, combined hemorrhages in both spinal compartments is discussed.
\end{abstract}

Keywords Spontaneous · Subarachnoid hemorrhage · Subdural hematoma $\cdot$ Spine $\cdot$ MRI

Y. Kakitsubata $\cdot$ Y. Miyata $\cdot$ Y. Ito $\cdot$ Y. Yuki

Department of Radiology, Miyazaki Social Insurance Hospital, Miyazaki, Japan

Y. Kakitsubata · S. J. Theodorou • D. J. Theodorou Department of Radiology, School of Medicine,

University of California, San Diego Medical Center,

San Diego, CA, USA

Y. Kakitsubata $\cdot$ S. J. Theodorou $\cdot$ D. J. Theodorou

Department of Radiology,

Veterans Administration Medical Center,

San Diego, CA, USA

\section{K. Honbu}

Department of Orthopedics, Miyazaki Social Insurance Hospital,

Miyazaki, Japan

T. Maehara

Department of Orthopedics, Maehara Hospital,

Kobayashi, Japan

D. J. Theodorou $(\square)$

13 Papadopoulos street,

Ioannina 45444, Greece

e-mail: daphne theodorou@hotmail.com

e-mail: rjtheodorou@hotmail.com

\section{Case report}

A 66-year-old man presented to the emergency department with history of 1 week of lower back pain, headache, and left lower limb pain. He had no history of trauma, physical exertion, anticoagulant therapy, or any known bleeding dyscrasias. The patient had a fever of $100^{\circ} \mathrm{F}$. He did not develop paraparesis, loss of sensation, numbness or urinary dysfunction. Physical examination disclosed localized tenderness over the lumbar spine. Neurologic examination was normal. Pertinent laboratory data were within normal limits except for mild elevation of lactate dehydrogenase (262 IU/1).

Urgent CT scan of the head showed no evidence of subarachnoid or intracerebral hemorrhage. MR imaging of the spine performed on the same day of admission demonstrated a linear subarachnoid lesion extending from L1 to L5, ventral to the conus medullaris and cauda equina. The subarachnoid process was of increased signal intensity relative to the spinal cord on both $\mathrm{T} 1$ - and T2-weighted images; which was suggestive of subacute hemorrhage (Fig. 1). In addition, a subdural lesion with a smooth contour was seen extending from T11 to T12 vertebrae and overlying the ventrolateral aspect of the spinal canal. This abnormal area displayed predominantly increased signal intensity relative to the spinal cord on T1-weighted images. On T2-weighted images, the lesion showed heterogeneously increased signal intensity with a focus of low signal intensity compatible with subacute hematoma containing deoxyhemoglobin (Fig. 2). The subdural hematoma was compressing the adjacent spinal cord without obvious signal changes within the cord. No MR-imaging evidence of vascular abnormalities was visualized in the spine. Although the patient was advised to undergo spinal angiography, he did not consent to. 

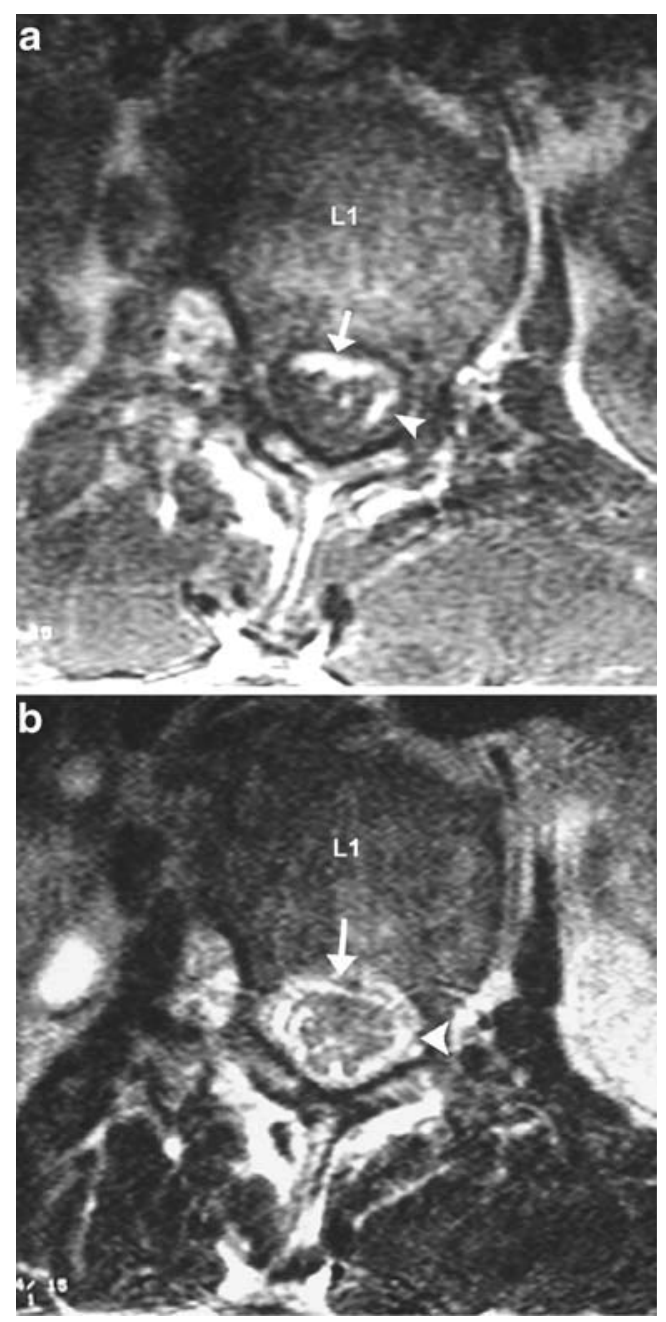

Fig. 1 a, b Axial T1- and T2-weighted MR images at the time of presentation 1 week after the onset of symptoms show subarachnoid hemorrhage of high signal intensity at the L1 level, in the anterior (arrow) and lateral (arrowhead) subarachnoid space

Lumbar puncture yielded bloody cerebrospinal fluid confirming subarachnoid hemorrhage. Since the patient was ambulatory and did not experience neurological deterioration, conservative management was opted. Repeat MR-imaging studies revealed gradual resolution of both hemorrhages (Fig. 3). Clinically, there was subsidence of symptoms over the following 2 weeks and the patient was discharged. At 5-month follow-up, the patient was asymptomatic and doing well.

\section{Discussion}

Most patients with spontaneous subarachnoid hemorrhage are found to have intracranial abnormalities [1]. Less than $1 \%$ of patients have a spinal source of subarachnoid hemorrhage that usually involves arteriovenous malformation or saccular aneurysm of a spinal artery. Occasionally, primary cord tumors (i.e., ependymoma, hemangioblastoma), nerve sheath tumors, or metastases are the source of subarachnoid hemorrhage. Spinal trauma, coagulopathies, and autoimmune vascular disorders are additional sources of hemorrhage in the subarachnoid space [2, 3]. Spontaneous spinal subarachnoid hemorrhage, however, is very rare. Further, the co-existence of spinal subarachnoid and subdural hemorrhage is extremely uncommon.

With regard to the pathogenesis of the condition, it has been suggested that minor trauma can increase both the intrathoracic and intraluminal pressure of spinal vessels, particularly the valveless radiculomedullary veins traversing the subdural and subarachnoid spaces. If the pressure of the cerebrospinal fluid (CSF) cannot neutralize this force, spinal vessels rupture resulting in subarachnoid hemorrhage [4]. Of note, the same increase in pressures provoked even by physical exertion can cause rupture of the small extraarachnoidal vessels located on the inner surface of the dura, resulting in subdural hemorrhage. Subdural hemorrhage may originate in the more vascular subarachnoid space and pass through rents of the thin and delicate arachnoid membrane. Conversely, the origin of hemorrhage can be the subdural space, where blood can pass through the arachnoid membrane, giving rise to an associated sub-
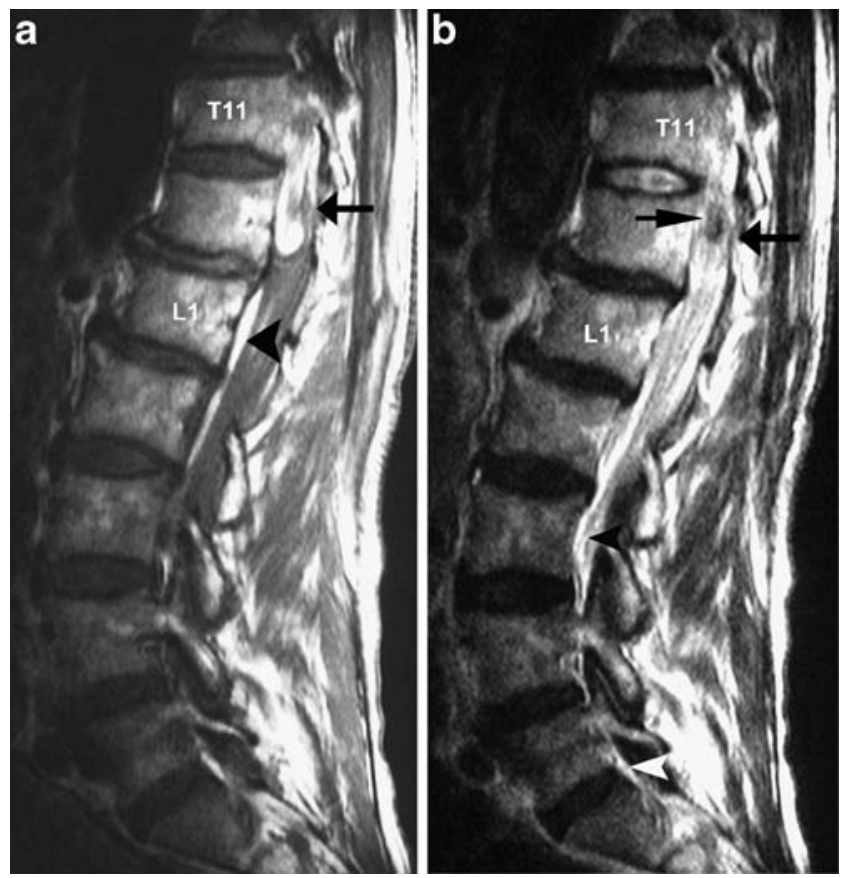

Fig. 2 a, b Corresponding sagittal T1- and T2-weighted MR images show increased signal in CSF extending from L1 through L5, consistent with subarachnoid hemorrhage (arrowheads). An ovoid area of high signal intensity on T1-weighted images (a) located at the T11-T12 level represents concomitant subdural hematoma (arrow). The hematoma (arrow), which is less well depicted on T2-weighted images (b) contains a characteristic focal region of low signal intensity (thin arrow) indicating the presence of deoxyhemoglobin 
Fig. 3 Evolutionary MRimaging changes of subdural hematoma show that as the hematoma aged, the signal intensity increased. a, b Followup axial T1- (a) and

T2-weighted (b) MR images obtained 26 days after onset of symptoms show subdural hematoma (arrow) of increased signal intensity on both sequences. c, d Repeat axial T1- (c) and T2-weighted (d) MR images at a 5-month follow-up show resolution of subdural hematoma
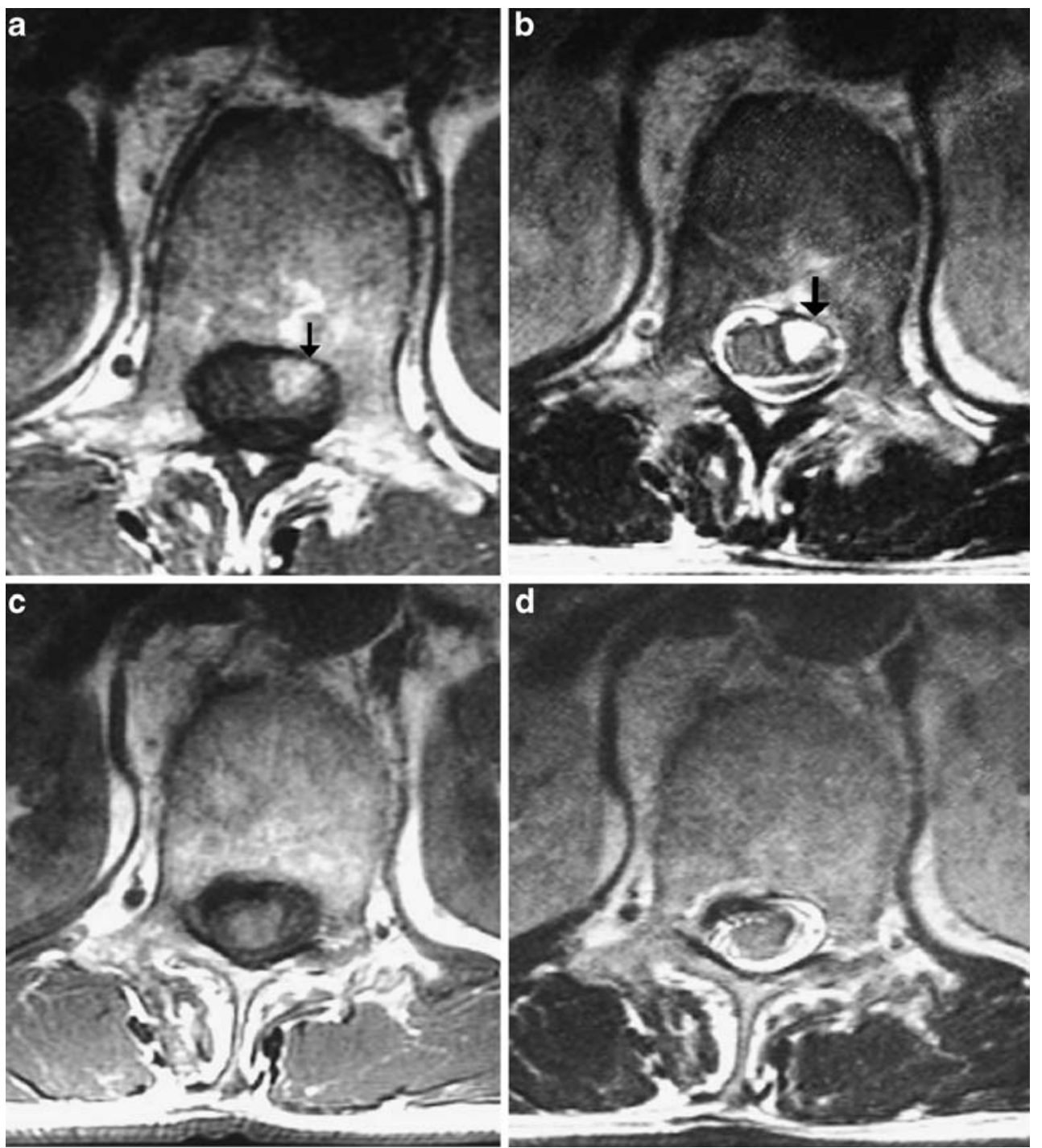

arachnoid hemorrhage. Indeed, it may be impossible to determine whether blood has its source in the subarachnoid or subdural compartment.

Spinal subarachnoid hemorrhage rarely presents as a hematoma owing to the diluting and redistributing effect of the CSF, unless the hematoma is sufficiently large to block the CSF flow [5]. Once subdural hematoma has formed, further subarachnoid bleeding may occur through partial block of the CSF flow. On the basis of clinical signs and symptoms, it may be difficult to distinguish between epidural, subdural, and subarachnoid spinal hemorrhage. All three forms may present with severe back pain, often of an acute onset, with or without sensorimotor deficits. Meningism, headache ( $70 \%$ of cases), and mental status changes $(22 \%$ of cases) are usually indicative of subarachnoid hemorrhage, however [2, 5]. Subarachnoid hemorrhage may have variable clinical presentation and prognosis according to its location within the spinal canal.
The dorsally located subarachnoid spinal hemorrhage most commonly causes thecal sac compression while the ventral type is usually not associated with neurological deterioration, and as such, surgical intervention may not be warranted. The presence of a spinal hematoma, however, causing thecal sac compression with rapidly progressive neurological sequela designates a neurosurgical emergency.

Spinal angiography is generally considered to be the gold standard for demonstrating spinal artery aneurysms, arteriovenous malformations, or other pathology that can cause spinal subarachnoid hemorrhage $[6,7]$. MR imaging is superb in the delineation of the extent of spinal hemorrhage and for determining the relationship between hemorrhage and the thecal sac [1]. It also remains the premier imaging modality for depicting the chronological changes of hemorrhage, facilitating monitoring of treatment. The MR signal intensity characteristics of hemorrhage are time-dependent. On T1-weighted images, an 
acute spinal hematoma less than 7 days old appears isointense, less commonly hyperintense, or hypointense to the spinal cord. On T2-weighted images, acute spinal hematomas are heterogeneous and hyperintense to the spinal cord with hypointense foci [8]. Low signal intensity seen on T2-weighted images is mainly due to the presence of deoxyhemoglobin [4]. Subacute lesions, more than 7 days but less than 1 month old, are often hyperintense and less commonly isointense to the spinal cord on T1weighted images. On T2-weighted images, subacute lesions demonstrate increased signal intensity characteristics. These signal characteristics are attributed to the effects of methemoglobin, which is strongly paramagnetic. Areas of spinal cord compression appear as high-signal-intensity regions on T2-weighted images.

Prompt identification of spinal hemorrhage is important for emergent treatment of patients and preservation of normal function. The prognosis varies with the severity of neurological impairment. Delay in diagnosis and treatment can easily cause irreversible and serious neurological deficit. Treatment focuses on surgical evacuation of hematoma with subsequent decompression of the thecal sac [5]. Alternatively, conservative therapy can be employed with close patient monitoring. Because our patient was ambulatory and did not experience neurological deterioration but instead gradual subsidence of his symptoms, we adopted a "wait and see" policy and careful conservative management was opted.
Open Access This article is distributed under the terms of the Creative Commons Attribution Noncommercial License which permits any noncommercial use, distribution, and reproduction in any medium, provided the original author(s) and source are credited.

\section{References}

1. Kim Y, Cho K, Chung C, Kim H (2004) Idiopathic spontaneous spinal subarachnoid hemorrhage. Case report. Spinal Cord 42:545547 doi: $10.1038 /$ sj.sc. 3101620

2. Ruelle A, Zerbi D, Andrioli G (2001) Spinal subarachnoid bleeding of unknown etiology. Case reports. J Neurosurg Sci 45:53-57

3. Vishteh A, Theodore N, Spetzler R (2000) Nontraumatic acute spinal subdural hematoma. J Neurosurg Spine 93:180-181

4. Morandi X, Riffaud L, Chabert E, Brassier G (2001) Acute nontraumatic spinal subdural hematomas in three patients. Spine 26(23):E547-E551 doi:10.1097/00007632-200112010-00022

5. Kyriakides A, Lalam R, El Masry W (2007) Acute spontaneous spinal subdural hematoma presenting as paraplegia. A rare case.. Spine 32(21):E619-E622

6. Massand M, Wallace R, Gonzalez L, Zabramski J, Spetzler R (2005) Subarachnoid hemorrhage due to isolated spinal artery aneurysm in four patients. AJNR Am J Neuroradiol 26:24152419

7. Berlis A, Scheufler K, Schmahl C, Rauer S, Gotz F, Schumacher M (2005) Solitary spinal artery aneurysms as a rare source of spinal subarachnoid hemorrhage: Potential etiology and treatment strategy. AJNR Am J Neuroradiol 26:405-410

8. De Wyngaert R, Ghysen D, Bellinck P, Mulkens T, Termote J (2007) Spontaneous spinal epidural hematoma. JBR-BTR 90:186187 